\title{
Análise da frequência anual das condições de céu em Botucatu, São Paulo
}

\author{
Érico T. Teramoto ${ }^{1}$ \& João F. Escobedo ${ }^{1}$
}

\begin{abstract}
RESUMO
Este trabalho apresenta uma análise da frequência das condições de céu em Botucatu. A classificação das condições de céu foi feita com base no índice de claridade $\left(K_{\mathrm{T}}\right)$, calculado a partir de uma base de dados de 10 anos de radiação global diária. Em Botucatu há, ao longo do ano, predominância de dias com condição de céu claro, em média 114 dias. Para a condição de céu nebuloso a frequência média é de 61 dias e, em referência às duas condições de céu intermediárias, a frequência de céu parcialmente nebuloso com dominância para o difuso, é de 94 dias e a com dominância para o claro é de 96 dias. Os principais fatores influentes sobre as condições de céu em Botucatu são as entradas de frentes frias e a formação da Zona de Convergência do Atlântico Sul, as quais respondem principalmente pela alteração da condição de céu claro para a de céu nebuloso ou para a de céu parcialmente nebuloso com dominância para o difuso. Os aerossóis resultantes das queimadas em lavouras de cana-de-açúcar respondem pela alteração da condição de céu claro para a de céu parcialmente nebuloso com dominância para o claro.
\end{abstract}

Palavras-chave: radiação solar, nebulosidade, índice de claridade

\section{Analysis of the annual frequency of the sky conditions in Botucatu, São Paulo}

\begin{abstract}
This paper presents an analysis of the frequency of the sky conditions in Botucatu. The classification of sky conditions was made based on the clearness index $\left(\mathrm{K}_{\mathrm{T}}\right)$, calculated from a database with 10 years of measured daily global solar radiation. Throughout the year in Botucatu, there is a predominance of days with clear sky condition, on average 114 days. For cloudy sky condition, the average frequency is 61 days. About the two intermediate sky conditions, the frequency of the sky partially cloudy with dominance for the diffuse is 94 days and the one with dominance for the clear is 96 days. The main influential factors on the sky conditions in Botucatu are the inputs of cold fronts and the formation of the South Atlantic Convergence Zone. They respond primarily by altering the sky condition of clear to cloudy or partially cloudy with dominance to diffuse. The aerosols resulting from burning in sugarcane fields respond by altering the sky condition from clear to partly cloudy with dominance for the clear.
\end{abstract}

Key words: solar radiation, cloudiness, clearness index 


\section{INTRODUÇÃO}

A radiação solar é uma das variáveis meteorológicas mais afetadas pela presença de nuvens sobre o céu. As nuvens são as principais atenuadoras ao absorver a radiação solar no comprimento de onda do infravermelho e ao refletir e espalhar grande parte da radiação solar no comprimento de onda do visível (Iqbal, 1983). As duas faixas espectrais correspondem, juntas, a aproximadamente $94 \%$ da radiação solar global (Escobedo et al., 2009). Por isto, em qualquer período do ano e local do globo terrestre os maiores níveis de incidência de radiação solar sobre a superfície terrestre ocorrem em condições de céu claro, ou céu sem nuvens, enquanto os menores são observados em condições de céu totalmente coberto por nuvens, ou céu nebuloso.

Assim, o estudo do padrão de variação das condições de céu permite entender a dinâmica da variação dos níveis de energia solar incidentes sobre a superfície terrestre e serve de parâmetro na estimativa da radiação solar, a partir de modelos estatísticos. Esses conhecimentos são úteis em projetos de sistemas que utilizem energia solar ou sejam influenciados por ela, em estudos voltados ao planejamento e desenvolvimento do turismo e na secagem de produtos agrícolas, entre outros (Souza et al., 2006; Pattanasethanon et al., 2007; Li et al., 2011).

A concentração e as características das nuvens sobre o céu variam fortemente em função do tempo e localização geográfica. Um dos fatores responsáveis pela alteração da nebulosidade é a variação na concentração de núcleos de condensação de nuvens (NCN) presentes na atmosfera. Parte dos aerossóis provenientes da queima de biomassa ou de combustíveis fósseis pode atuar como NCN ou como seus precursores, sendo capazes de alterar as propriedades físicas e o tempo de vida das nuvens (Wielicki et al., 2005; Kaufmann \& Koren, 2006; Kvalevag \& Myhre, 2007; Codato et al., 2008). Li \& Lam (2000) em Hong Kong, Ogunjobi et al. (2002) em Kwangju e Seoul na Coréia do Sul e Che et al. (2006) observaram, em várias cidades na China, baixas frequências da condição de céu claro em condições de elevada concentração de aerossóis na atmosfera. A alteração da nebulosidade nesses casos pode estar relacionada ao aumento na concentração de NCN na atmosfera. Algumas dessas cidades possuem profundidade ótica de aerossóis (AOD) média anual, entre 0,37 e 0,65 (Luo et al., 2000; Cheng et al., 2006).

Este trabalho objetivou apresentar uma análise climática da frequência das condições de céu em Botucatu. Todos os anos a atmosfera da região de Botucatu recebe, durante o outono e o inverno austrais, elevadas quantidades de aerossóis resultantes das queimadas em lavouras de cana-de-açúcar de várias cidades adjacentes, sendo ainda desconhecidos os efeitos desta alteração ambiental sobre as condições de céu no município.

\section{Material e MÉTODOS}

Botucatu está situada na região centro-sul do estado de São Paulo (latitude $22,85^{\circ} \mathrm{S}$, longitude $48,43^{\circ} \mathrm{W}$, altitude 786 $\mathrm{m})$, distante $221 \mathrm{~km}$ do Oceano Atlântico, inserida em uma região rural caracterizada pela produção de cana-de-açúcar.
O clima do município é caracterizado por um inverno frio e seco e verão quente e úmido (Cunha \& Martins, 2009). Os meses de fevereiro e julho são, respectivamente, o mais quente e o mais frio do ano, com temperaturas médias de 23,21 e 17,05 ${ }^{\circ} \mathrm{C}$ (Figura 1); enquanto os meses de fevereiro e agosto são o mais e o menos úmido, com percentuais de 77,07 e 61,72\%, respectivamente.

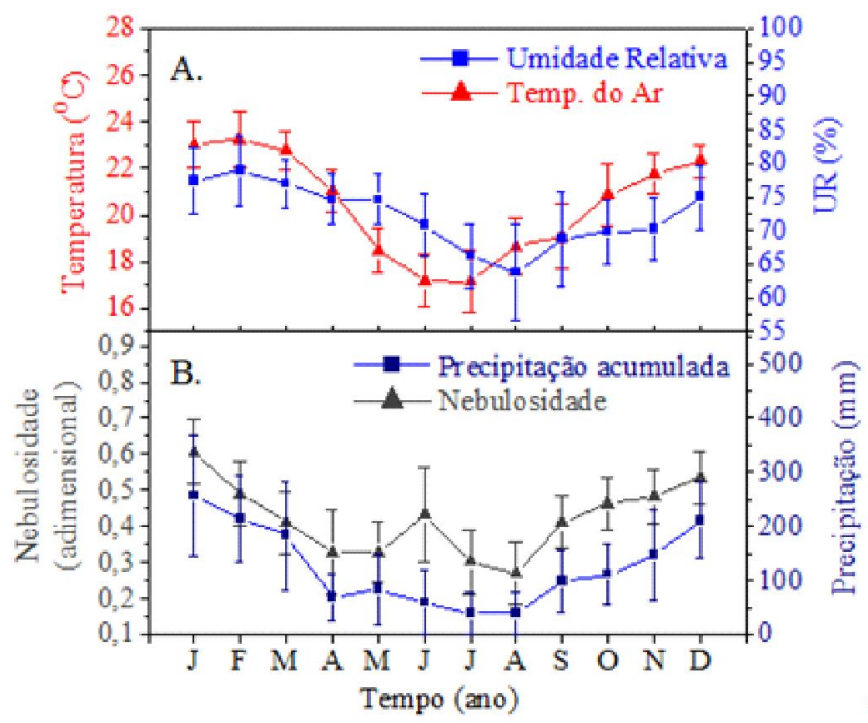

Figura 1. Médias diárias mensais dos parâmetros meteorológicos (A) Temperatura do ar, umidade relativa, (B) nebulosidade e precipitação em Botucatu, período de 1971 a 2000

O ciclo anual da chuva é constituído de dois períodos bem distintos: um período chuvoso (outubro a março), quando ocorrem de 75 a $90 \%$ do total anual de precipitação acumulada, e um período seco (abril a setembro), em que os índices pluviométricos se encontram abaixo do nível de $100 \mathrm{~mm}$, em que no primeiro a ocorrência de chuvas e a elevada nebulosidade são resultantes da formação da Zona de Convergência do Atlântico Sul e dos Sistemas Frontais (Carvalho et al., 2004; CPTEC, 2010; Reboita et al., 2010) e no segundo, há baixa nebulosidade e as chuvas que ocorrem são de média a pequena intensidade e resultantes da passagem de frentes frias (CPTEC, 2010). O maior valor de precipitação acumulada ocorre no mês de janeiro $(288,22 \mathrm{~mm})$, quando a razão de nebulosidade é 0,61 , e o menor em agosto (40,69 mm), em que a razão de nebulosidade é 0,27 . A razão de nebulosidade aqui citada, expressa o número de horas em que o Sol ficou encoberto por nuvens, numa razão de insolação $n / \mathrm{N}$, sendo $n$ o número de horas de brilho solar e $\mathrm{N}$ o fotoperíodo, sendo definida pela Eq. 1:

$$
\varphi=1-\left(\frac{\mathrm{n}}{\mathrm{N}}\right)
$$

O critério usado neste trabalho para classificar a condição de céu foi o desenvolvido por Escobedo et al. (2009) para Botucatu, São Paulo, o qual classificou as condições de céu em 4 tipos em função de $\mathrm{K}_{\mathrm{T}}$. Esta metodologia dispensa o uso 
de dados de radiação direta e/ou difusa, que são medidas como rotina em poucas estações meteorológicas brasileiras, e consideram os seguintes tipos de condição de céu (Figura 2).

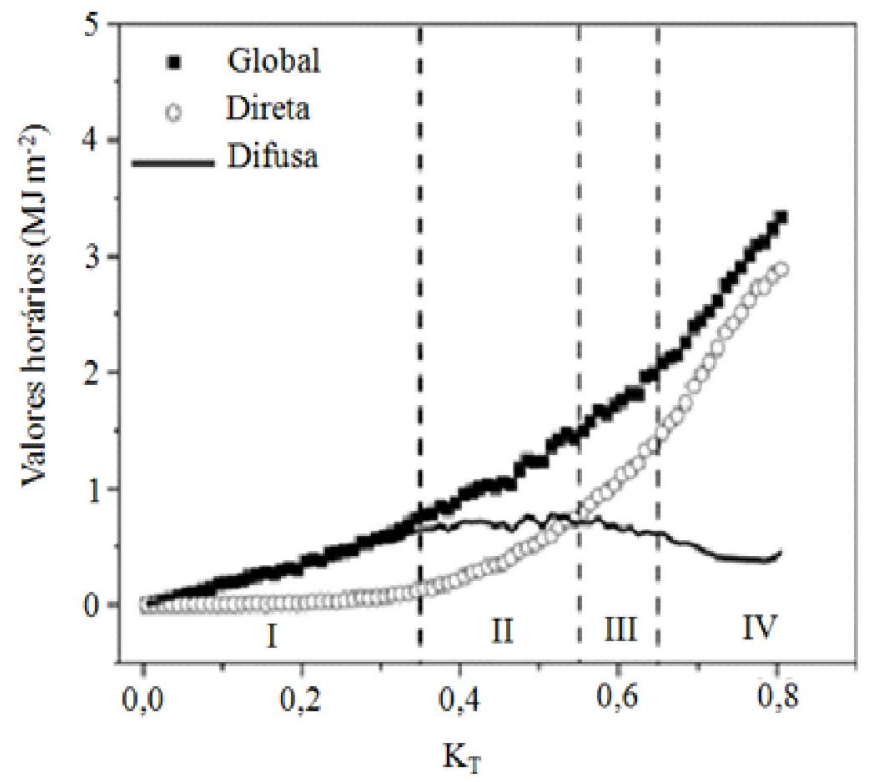

Fonte: Escobedo et al. (2009)

Figura 2. Evolução das irradiações global, difusa e direta na horizontal em função de intervalos infinitesimais de $\mathrm{K}_{\mathrm{T}}$

Condição de céu $\mathrm{I}$, nebuloso: $\mathrm{K}_{\mathrm{T}} \leq 0,35$, a radiação direta é praticamente igual a zero, ou seja, a radiação global é composta apenas pela radiação difusa;

Condição de céu II, parcialmente nebuloso com dominância para o difuso: $0,35<\mathrm{K}_{\mathrm{T}} \leq 0,55$, a radiação global é composta de uma parcela maior da radiação difusa, que decresce variando gradativamente entre 88 a 50\% da radiação global;

Condição de céu III, parcialmente nebuloso com dominância para o claro: $0,55<\mathrm{K}_{\mathrm{T}} \leq 0,65$, a radiação global é composta de uma parcela maior da radiação direta, que aumenta gradativamente enquanto a radiação difusa diminui variando entre 50 a $30 \%$ da radiação global;

Condição de céu IV, claro: $\mathrm{K}_{\mathrm{T}}>0,65$, a radiação global é composta predominantemente pela radiação direta. 1983):

$\mathrm{O}$ índice de claridade $\mathrm{K}_{\mathrm{T}}$ foi calculado através da Eq. 2 (Iqbal,

$$
\mathrm{K}_{\mathrm{T}}=\frac{\mathrm{H}_{\mathrm{G}}}{\mathrm{H}_{0}}
$$

donde:

$\mathrm{H}_{\mathrm{G}}$ - radiação global na horizontal diária, $\mathrm{MJ} \mathrm{m}^{-2} \mathrm{~d}^{-1}$

$\mathrm{H}_{0}$ - radiação solar no topo da atmosfera diária, $\mathrm{MJ} \mathrm{m}^{-2} \mathrm{~d}^{-1}$

A base de dados de $\mathrm{H}_{\mathrm{G}}$ diária foi medida por um piranômetro Eppley PSP da Campbell Scientifc, instalado na Estação Radiométrica da Faculdade de Ciências Agronômicas da UNESP em Botucatu, São Paulo, Brasil, no período de janeiro de 1996 a dezembro de 2005. Esta base de dados possui medições de 95\% do período entre 1996 a 2005. A base de dados de $\mathrm{H}_{0}$ diária foi estimada a partir da Eq. 3 (Iqbal, 1983):

$$
\begin{aligned}
\mathrm{H}_{0} & =1367\left(\frac{24}{\pi}\right) \mathrm{E}_{0}\left[\left(\frac{\pi}{180}\right) \omega_{\mathrm{s}} \operatorname{sen}(\varphi) \operatorname{sen}(\delta)+\right. \\
& \left.+\cos (\varphi) \cos (\delta) \operatorname{sen}\left(\omega_{\mathrm{s}}\right)\right]
\end{aligned}
$$

em que:

$\mathrm{E}_{0}$ - fator de correção da excentricidade da órbita terrestre

$\omega_{\mathrm{s}}$ - ângulo horário solar, ${ }^{\mathrm{o}}$

$\varphi \quad$ - latitude local, ${ }^{\circ}$

$\delta$ - declinação solar, ${ }^{\circ}$

O fator de correção da excentricidade da órbita terrestre, a declinação solar e o ângulo horário solar foram calculados, respectivamente, a partir das seguintes equações:

$$
\begin{array}{r}
\mathrm{E}_{0}=1,000+0,034 \cos (\Gamma)+0,0013 \operatorname{sen}(\Gamma) \\
+\quad 0,000719 \cos (2 \Gamma)+0,000077 \cos (2 \Gamma) \\
\delta=23,45 \sin \left[\left(\frac{360}{365}\right)(\mathrm{DJ}+284)\right] \\
\omega_{\mathrm{s}}=\operatorname{arcos}[-\operatorname{tg}(\phi) \operatorname{tg}(\delta)]
\end{array}
$$

donde:

$$
\begin{aligned}
& \text { DJ - dia Juliano } \\
& \Gamma \quad-2 \pi(\text { DJ }-1) / 366
\end{aligned}
$$

Neste trabalho também foram utilizadas bases de dados diários de insolação, precipitação pluviométrica e profundidade óptica de aerossóis. Os dados de insolação e de precipitação pluviométrica foram medidos, respectivamente, por um heliógrafo da Campbell-Strokes e por um pluviógrafo da Ota Keiki Seisakusho no período de janeiro de 1996 a dezembro de 2005. Os dois instrumentos estão instalados na Estação Climatológica da Faculdade de Ciências Agronômicas da UNESP, em Botucatu, São Paulo, Brasil. Os dados de profundidade óptica de aerossóis (AOD) foram medidos pelo satélite TERRA, no período de janeiro de 2000 a dezembro de 2005, disponíveis no site http://terra.nasa.gov. A medição foi feita através do instrumento MODIS (Moderate Resolution Imaging Spectroradiometer) na banda 4, de 0,55 $\mu \mathrm{m}$. Este satélite entrou em atividade em dezembro de 1999 e faz parte do programa EOS (Earth Observing System) da NASA (2010).

As bases de dados de insolação e de precipitação pluviométrica foram sincronizadas com as de radiação global apresentando, assim, medições de 95\% do período entre 1996 a 2005. A base de dados de AOD não foi sincronizada à de radiação global e possui medições de $71 \%$ do período entre 2000 a 2005.

Visando à determinação das frequências anuais das condições de céu, a partir dos valores de $\mathrm{K}_{\mathrm{T}}$ calculados, classificaram-se os dias da base total de dados em condição de céu I, II, III ou IV; em seguida, obteve-se a distribuição de frequência para as quatro classes na escala de tempo anual.

Os dados de frequência anual de cada uma das quatro condições de céu foram submetidos ao teste W de ShapiroWilk para verificar a normalidade a nível de significância de 5\% de probabilidade (Shapiro \& Wilk, 1965). Este teste não- 
paramétrico é robusto e recomendado para verificação da normalidade quando o número de repetições é menor que 30. A hipótese nula do teste é que os dados provêm de uma população normal e ela é aceita quando $\mathrm{W}>\mathrm{W}_{\mathrm{c}}$, sendo $\mathrm{W}_{\mathrm{c}}$ tabelado e $\mathrm{W}$ determinado pela equação:

$$
\mathrm{W}=\frac{\left(\sum_{\mathrm{i}=1}^{\mathrm{n}} \mathrm{a}_{\mathrm{i}} \mathrm{x}_{\mathrm{i}}\right)}{\sum_{\mathrm{i}=1}^{\mathrm{n}}\left(\mathrm{x}_{\mathrm{i}}-\overline{\mathrm{x}}\right)^{2}}
$$

donde:

$$
\begin{aligned}
& n-\text { número de amostras } \\
& x_{1}-\text { valores observados ordenados } \\
& x_{1} \text { - média } \\
& a_{i} \text { - coeficientes de Shapiro-Wilk (tabelados) }
\end{aligned}
$$

Também foi analisada a simetria através do coeficiente de assimetria "Skewness" (AS) dividido pelo erro padrão (se). O coeficiente de assimetria AS foi calculado por meio de:

$$
\text { AS }=\frac{1}{(n-1) \delta^{3}} \sum_{i=1}^{n}\left(x_{i}-\bar{x}\right)^{3}
$$

donde:

$$
\begin{array}{ll}
n & \text { - número de amostras } \\
X_{1} & \text { - valores observados } \\
X & \text { - média } \\
\delta & \text { - desvio padrão }
\end{array}
$$

Resultados da razão AS/se entre 2 e -2 indicam que a distribuição é simétrica. A confirmação da normalidade e da simetria indica a possibilidade do uso da distribuição z.

Para analisar a série climatologica de 10 anos de frequência das condições de céu, recorreu-se à estatistica descritiva usual e à distribuição z. A distribuição z foi utilizada para analisar as variações interanuais dos valores de frequência. Nesta análise, qualquer valor de $\mathrm{z}$ fora do intervalo $-1,65>\mathrm{z}>1,65$ foi considerado alteração anormal na frequência anual, ou seja, valores com probabilidade de ocorrência menor que $10 \%$.

\section{RESULTADOS E DISCUSSÃO}

\section{Variação interanual da radiação global e do índice de claridade $\mathbf{K}_{\mathbf{T}}$}

Apresenta-se, na Figura 3 a evolução dos valores de radiação global média anual diária $\left(\overline{\mathrm{H}}_{\mathrm{G}}\right)$ em Botucatu, no período de 1996 a 2005; nela, o intervalo entre as duas linhas tracejadas representa os valores de radiação global previstos pela média da série climatológica de 10 anos.

Os valores de $\overline{\mathrm{H}}_{\mathrm{G}}$ variaram de $16,72 \mathrm{MJ} \mathrm{m}^{-2} \mathrm{~d}^{-1}$ em $1998 \mathrm{a}$ $18,32 \mathrm{MJ} \mathrm{m}^{-2} \mathrm{~d}^{-1} \mathrm{em} 1999$, sendo a média da série climatológica de 10 anos equivalente a $17,54 \pm 0,51 \mathrm{MJ} \mathrm{m}^{-2} \mathrm{~d}^{-1}$. As variações interanuais nos valores de $\overline{\mathrm{H}}_{\mathrm{G}}$, observadas na Figura 3, são resultantes, basicamente, da ocorrência de diferentes condições de nebulosidade e concentração de vapor de água na atmosfera, no mesmo período. Os anos de 1998, 1999 e 2001 apresentaram

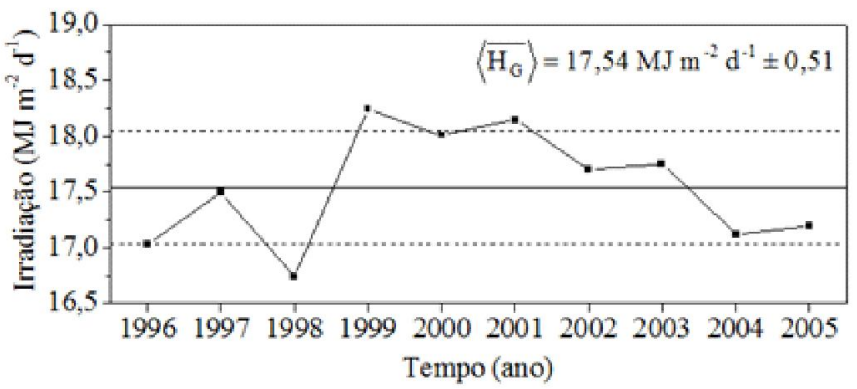

Figura 3. Evolução da radiação global média diária anual no período de 1996 a 2005

valores de $\overline{\mathrm{H}}_{\mathrm{G}}$ fora do intervalo de previsão da média da série climatológica de 10 anos; em 1999 e 2001, os valores foram superiores à média da série $\left(18,32\right.$ e $18,15 \mathrm{MJ} \mathrm{m}^{-2} \mathrm{~d}^{-1}$, respectivamente), enquanto o ano de 1998 apresentou valor inferior $\left(16,72 \mathrm{MJ} \mathrm{m}^{-2} \mathrm{~d}^{-1}\right)$.

Na Figura 4 são apresentadas a média anual do índice de nebulosidade $(\bar{\varphi})$, a frequência anual de dias com ocorrência de chuva $\left(\overline{f_{c}}\right)$ e a precipitação acumulada anual $(\mathrm{P})$ no período de 1996 a 2005.

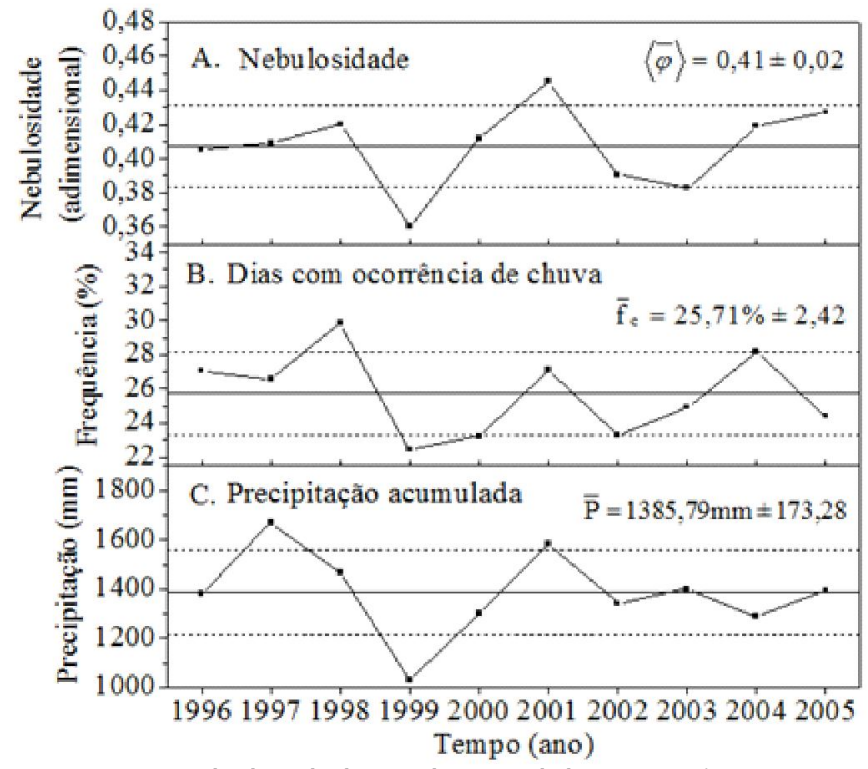

Figura 4. Nebulosidade média anual diária (A), frequência anual de dias com ocorrência de chuva (B) e Precipitação acumulada anual (C) no período de 1996 a 2005

Através da Figura 4A verifica-se que o aumento no valor de $\overline{\mathrm{H}}_{\mathrm{G}}$ observado em 1999 foi resultante das condições atmosféricas de baixa nebulosidade $(\bar{\varphi}=0,36)$; os baixos valores de frequência de ocorrência de chuva $\left(\bar{f}_{c}=22,47 \%\right)$ e precipitação acumulada $(\mathrm{P}=1030,5 \mathrm{~mm})$ também evidenciam que o ano foi seco, ou seja, apresentou baixa concentração de vapor de água na atmosfera.

Diferente de 1999, em 2001 o aumento de $\overline{\mathrm{H}}_{\mathrm{G}}$ ocorreu em condições atmosféricas de elevada nebulosidade $(\bar{\varphi}=0,45)$ resultado este decorrente de alterações sazonais ocultadas pela média anual. Climatologicamente, em Botucatu o verão austral apresenta elevadas nebulosidade e umidade relativa do ar (Figura 1), porém é também a estação na qual o Sol declina próximo da 
latitude local e por isso os valores de $\mathrm{H}_{\mathrm{G}}$ são os maiores do ano; caso contrário, no inverno austral há baixas nebulosidade e umidade relativa do ar e os níveis de energia de $\mathrm{H}_{\mathrm{G}}$ são os menores do ano pois é quando o Sol declina mais distante da latitude local. Em 2001 o verão foi relativamente seco e com baixa nebulosidade, condições atmosféricas que geraram um acentuado aumento nos valores $\mathrm{de}_{\mathrm{G}}$ neste período e o inverno foi úmido e nebuloso, com baixos valores de $\mathrm{H}_{\mathrm{G}}$. No cálculo da média anual esta inversão de condições atmosféricas entre o verão e o inverno gerou não só o aumento no valor de $\overline{\mathrm{H}}_{\mathrm{G}}$ mas também no de $\bar{\varphi}$, ou seja, dentro da média anual a nebulosidade elevada durante o inverno compensou, de maneira mais ou menos proporcional, a redução ocorrida durante o inverno; no entanto, como os níveis de $\mathrm{H}_{\mathrm{G}}$ são bastante elevados durante o verão se comparados ao inverno, a atenuação ocorrida durante o inverno nebuloso foi inferior ao aumento verificado durante o verão, com pouca nebulosidade.

No ano de 1998 a redução no valor de $\overline{\mathrm{H}}_{\mathrm{G}}$ em referência aos demais anos, foi resultante do aumento na concentração de vapor de água presente na atmosfera. $\mathrm{O}$ valor de frequência de ocorrência de chuva neste ano foi o maior da série climatológica de $10 \operatorname{anos}\left(\overline{f_{c}}=29,83 \%\right)$ e dos parâmetros climáticos nebulosidade e precipitação acumulada que foram relativamente altos $(\bar{\varphi}=0,42 ; \mathrm{P}=1467,50 \mathrm{~mm})$.

Com relação à transmissividade atmosférica da radiação global ao longo dos 10 anos, na Figura 5 é apresentada a variação do índice de claridade $\mathrm{K}_{\mathrm{T}}$ médio anual diário $\left(\overline{\mathrm{K}}_{\mathrm{T}}\right)$.

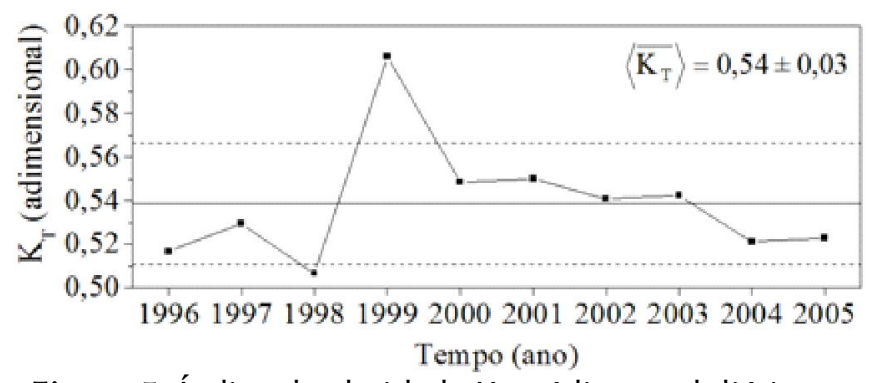

Figura 5. Índice de claridade $\mathrm{K}_{\mathrm{T}}$ médio anual diário no período de 1996 a 2005

O comportamento da curva dos valores de $\overline{\mathrm{K}}_{\mathrm{T}}$ (Figura 5) é similar ao da curva de $\overline{\mathrm{H}}_{\mathrm{G}}$ (Figura 3 ). Os valores de $\overline{\mathrm{K}}_{\mathrm{T}}$ variaram de 0,56 em 1999 a 0,51 em 1998, quando ambos os valores extrapolaram o intervalo de previsão da média da série climatológica de dez $\operatorname{anos}\left(\overline{\mathrm{K}}_{\mathrm{T}}=0,54 \pm 0,03\right)$. A elevada transmissividade ocorrida em 1999 foi resultante das condições atmosféricas de baixa nebulosidade enquanto a baixa transmissividade foi, em 1998, resultante da elevada concentração de vapor de água e de nuvens, conforme discutido anteriormente para $\overline{\mathrm{H}}_{\mathrm{G}}$.

Foi nessas condições de transmissividade atmosférica e de níveis de energia de radiação global que se estabeleceu a distribuição de frequência das quatro condições de céu, discutidas no item a seguir.

\section{Frequência anual das condições de céu}

Na Figura 6 são apresentados os valores de frequência anual diária das quatro condições de céu ao longo do período de 1996 a 2005.

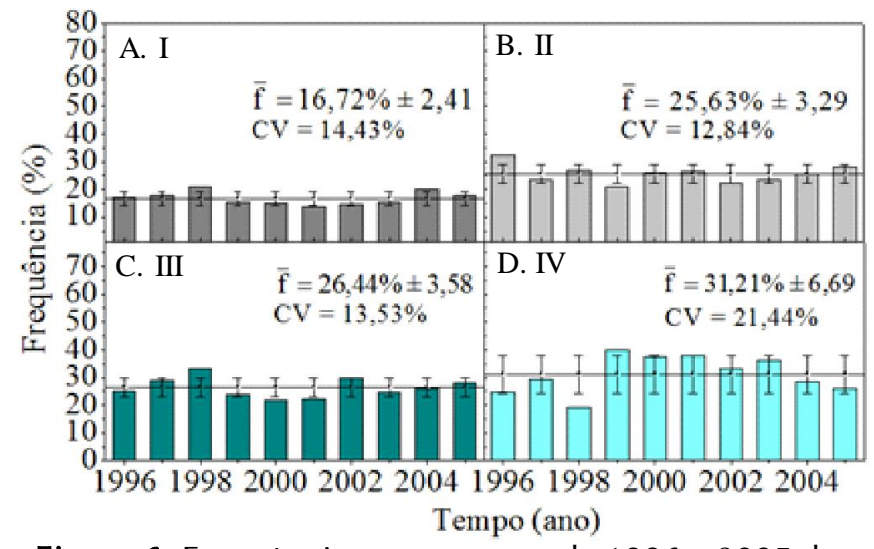

Figura 6. Frequência entre os anos de 1996 a 2005 das condições de céu I (A), II (B), III (C) e IV (D)

Conforme a Figura 6A, verifica-se que em Botucatu há baixa frequência de dias com ocorrência de céu nebuloso, quando comparada com demais condições de céu; ao longo dos 10 anos os valores de frequência da condição de céu I variaram de $13,68 \%$ em 2001 a 20,78\% em 1998, com média da série climatológica $\overline{\mathrm{f}}=16,72 \% \pm 2,41$. Por outro lado, a condição de céu claro é predominante ao longo do ano (Figura 6D), cuja frequência média da série climatológica é $\overline{\mathrm{f}}=31,21 \% \pm 6,69$ é, também, a condição de céu que apresentou a maior amplitude de variação ao longo dos 10 anos; enfim, os valores de frequência da condição de céu IV variaram de 24,89\% em 2005 a 39,81\% em 1999.

Em relação às duas condições de céu intermediárias (II e III), a frequência da II (Figura 6B) variou de 20,90\% em 1998 a $32,56 \%$ em 1996, com média da série climatológica $\overline{\mathrm{f}}=25,63 \% \pm$ 3,29 e a da III (Figura 6C) oscilou de 21,88\% em 2000 a 33,13\% em 1998, com média da série climatológica $\overline{\mathrm{f}}=26,44 \% \pm 3,48$. A comparação entre a soma da frequência das condições de céu I e II $(42,35 \%)$ com a das III e IV $(57,65 \%)$ evidencia que, na maioria dos dias do ano em Botucatu, a radiação global é composta por uma parcela maior de radiação direta, em relação à difusa.

\section{Impacto das queimadas em lavouras de cana-de-açúcar sobre as condições de céu}

Em relação às prováveis influências dos aerossóis sobre as condições de céu, partículas de aerossóis podem atuar como núcleos de condensação de nuvens e alterar a capacidade de elas absorverem, espalhar e refletir a radiação direta (Wielicki et al., 2005; Kaufmann \& Koren, 2006). Os aerossóis também são capazes de espalhar e absorver a radiação direta reduzindo os níveis de radiação global sem alteração da nebulosidade (Lohmann \& Feichter, 2005; Kvalevag \& Myhre, 2007; Koren et al., 2008); por outro lado, o aumento na concentração de vapor de água na atmosfera pode alterar o tamanho das partículas de aerossóis, alterando suas propriedades óticas e aumentando a atenuação da radiação global (Codato et al., 2008). A redução nos níveis de radiação global gera redução nos valores de $\mathrm{K}_{\mathrm{T}}$, podendo alterar a condição de céu de claro para outras intermediárias. Portanto, variações nas concentrações de aerossóis na atmosfera normalmente são acompanhadas por reduções na frequência das condições de 
céu claro e aumento da componente difusa dentro da radiação global.

O aumento na concentração de aerossóis na atmosfera sobre Botucatu ocorre durante o período seco do ano, quando são baixas a umidade relativa do ar e a concentração de nuvens, condições em que há fraca atenuação da radiação global e a redução na frequência da condição de céu IV pode ser acompanhada de aumento na de céu III.

Na Figura 7A são apresentadas as evoluções dos valores médios de frequência anual diária das condições de céu III e IV e a dos valores médios anuais diários de AOD em Botucatu, no período de 2000 a 2005; na 7B é apresentado o diagrama de dispersão da relação entre a profundidade ótica de aerossóis e a frequência das condições de céu III.

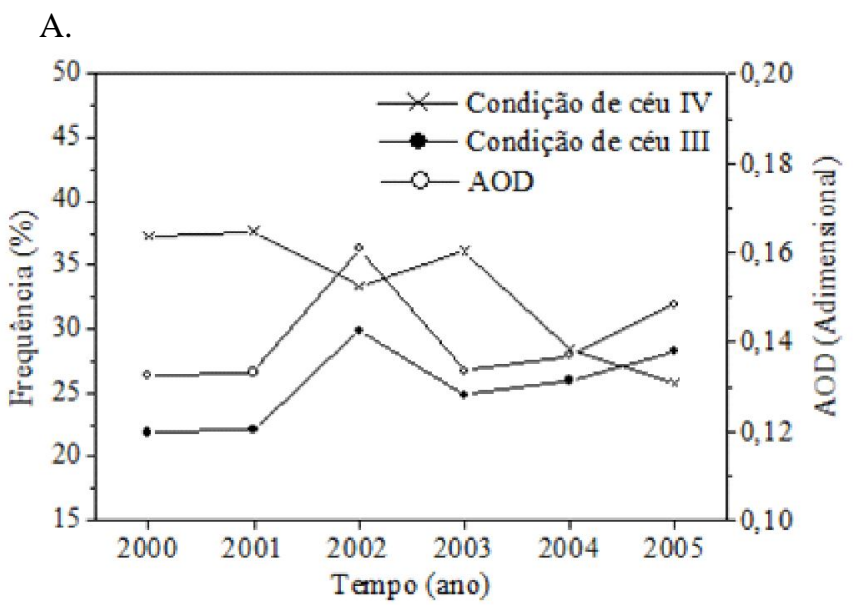

B.

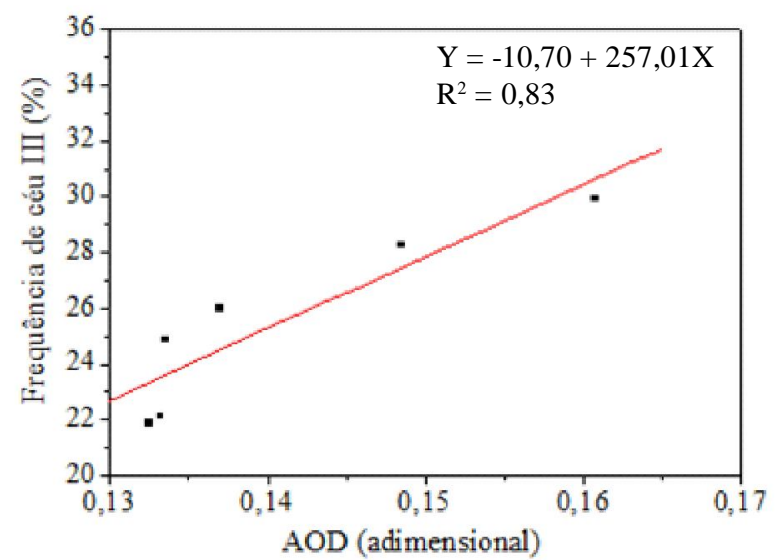

Figura 7. Frequência anual das condições de céu III e IV e profundidade ótica de aerossóis (AOD) média anual diária no período de 2000 a 2005

Conforme a Figura 7A, a curva dos valores médios de frequência da condição de céu IV apresenta comportamento inverso ao das curvas de valores médios de AOD e de frequência da condição de céu III. Verifica-se que aumentos na concentração de aerossóis são acompanhados de reduções na frequência da condição de céu claro, com consequente aumento na frequência da condição de céu parcialmente nebuloso, com dominância para o claro. Aproximadamente $83 \%$ das variações na frequência da condição de III estão relacionados a variações na concentração de aerossóis na atmosfera sobre Botucatu (Figura 7B).
A não proporcionalidade na variação da frequência da condição de céu claro em relação à variação na concentração de aerossóis, resulta da influência de outros fatores. A formação da Zona de Convergência do Atlântico Sul (ZCAS) e dos Sistemas Frontais (SF) é exemplo desses fatores; juntos, tais sistemas sinóticos são os principais determinantes do padrão de variação das condições de céu, em grande parte do estado de São Paulo (CPTEC, 2010; Reboita et al., 2010). Respondem pela redução da condição de céu IV para a de céu I, II ou III, através do aumento da nebulosidade e da concentração de vapor de água na atmosfera. A ZCAS é caracterizada como banda persistente de nebulosidade, que se estende desde o sul da Amazônia até o Atlântico Sul-Central e gera a ocorrência de chuvas intensas e persistentes durante o verão austral (Carvalho et al., 2004). Os SF, resultantes principalmente da passagem de frentes frias, geram aumento da nebulosidade e/ ou ocorrência de chuvas de baixa a média intensidade durante todo o ano porém com maior frequência durante o outono e a primavera austrais (CPTEC, 2010).

É possível haver interação entre o efeito das queimadas em lavouras de cana-de-açúcar, aumentando a concentração de NCN na atmosfera, com o efeito dos SF aumentando a concentração de vapor de água na atmosfera. O aumento da umidade do ar em condições de elevada concentração de NCN causaria uma distribuição maior do vapor de água disponível entre as partículas de aerossóis. Nessas condições as gotículas formadas crescem pouco em volume e lentamente, não favorecendo a ocorrência de precipitação; as nuvens formadas permaneceriam mais tempo cobrindo o céu e reduzindo a frequência das condições de céu IV ou III, antes de evaporarem ou ser carregada por correntes convectivas para outros locais; entretanto, a alteração na frequência das condições de céu por esta interação não é perceptível em escala anual de tempo.

\section{Variação interanual da frequência das condições de céu}

Sendo $\mathrm{W}_{\mathrm{c}}=0,842$ para amostras com $n=10$, verifica-se que os valores de frequência de cada uma das quatro condições de céu seguem distribuição normal simétrica, conforme Tabela 1, abaixo.

Tabela 1. Valores de W e AS/se calculados para os dados de frequência anual de cada uma das quatro condições de céu

\begin{tabular}{ccccc}
\hline \multirow{2}{*}{ Índice } & \multicolumn{4}{c}{ Condição de céu } \\
\cline { 2 - 5 } & I & II & III & IV \\
AS/se & 0,530 & 0,580 & 0,360 & 0,200 \\
W & 0,929 & 0,952 & 0,957 & 0,947 \\
\hline
\end{tabular}

Na Figura 8 se apresentam os valores de $\mathrm{z}$ das quatro condições de céu em Botucatu, no período de 1996 a 2005.

Conforme a Figura 8 ocorreu alteração anormal em pelo menos uma das condições de céu em 1996 e em 1998. Em 1996 houve aumento anormal na frequência da condição de céu II e em 1998 aumentos na da I e da III e redução anormal na da IV.

A ausência de linearidade na frequência de ocorrência anual e no tempo de atuação da ZCAS e dos SF é o principal determinante das variações interanuais na frequência das quatro condições de céu. Ocorrem, em média, 55 eventos de SF 


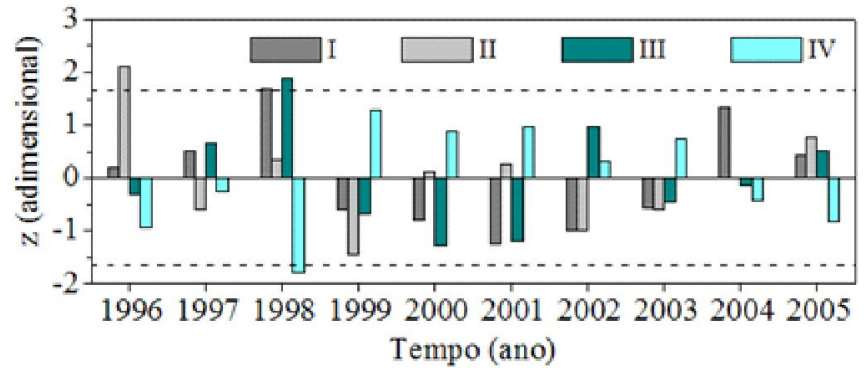

Figura 8. Valores de $z$ referentes à frequência das condições de céu I, II, III e IV entre os anos de 1996 a 2005

por ano entre as latitudes de 25 a $20^{\circ} \mathrm{S}$, com tempo de atuação variando de 1 a 3 dias cada um (CPTEC, 2010). A frequência média de ocorrência da ZCAS, com influência sobre o estado de São Paulo, é de 4 eventos ao ano com duração de até 11 dias (CPTEC, 2010).

Em 1996 houve um elevado aumento na frequência da condição de céu II, quando apresentou valor com probabilidade de ocorrência menor que $1,7 \%(z=2,11)$. Este aumento foi acompanhado de pequenas reduções na frequência das condições de céu III e IV. Neste ano a frequência de ocorrência dos SF e da ZCAS esteve dentro da média. Ocorreram 52 eventos de SF entre as latitudes de 25 a $20{ }^{\circ} \mathrm{S}$ e 4 eventos de ZCAS (CPTEC, 2010).

As alterações anormais na frequência das condições de céu I $(z=1,68)$, III $(z=1,87)$ e IV $(z=-1,78)$ observadas no ano de 1998 , foram resultantes do aumento na frequência de ocorrência dos SF. Segundo CPTEC (2010), em 1998 ocorreram 66 eventos de sistemas frontais entre as latitudes de 25 a $20^{\circ} \mathrm{S}$; para a ZCAS, a frequência de ocorrência no ano de 1998 foi de 2 eventos (CPTEC, 2010).

\section{CONClusÕES}

1. Em Botucatu há predominância de condições atmosféricas de baixa nebulosidade, com elevada frequência de dias com condição de céu claro e baixa de céu nebuloso.

2. Há indicativos da existência de influência dos aerossóis oriundos da queima de cana-de-açúcar sobre as condições de céu em Botucatu, reduzindo a frequência da condição de céu claro.

\section{LITERATURA CITADA}

Aguiar, D. A.; Rudorff, B. F. T.; Adami, M.; Shimabukuro, Y. E. Imagens de sensoriamento remoto no monitoramento da colheita da cana-de-açúcar. Engenharia Agrícola, v.29, p.440$451,2009$.

Calbó, J.; González, J. A.; Pagés, D. A method for sky-condition classification from ground-based solar radiation measurements. Journal of Applied Meteorology, v.40, p.21932199, 2001.

Carvalho, L. M. V.; Jones, C.; Liebmann, B. The South Atlantic Convergence Zone: Intensity, Form, Persistence, and Relationships with Intraseasonal to Interannual Activity and Extreme Rainfall. Journal of Climate, v.17, p.88-108, 2004.
Che, H. Z.; Shi, G. Y.; Zhang, X. Y; Zhao, J. Q.; Li, Y. Analysis of sky conditions using 40 year records of solar radiation data in China. Theory and Applied Climatology, v.89, p.83-94, 2006.

Cheng, A. Y. S.; Chan, M. H.; Yang, X. Study of aerosol optical thickness in Hong Kong, validation, results, and dependence on meteorological parameters. Atmospheric Environment, v.40, p.4469-4477, 2006.

Codato, G.; Oliveira, A. P.; Soares, J.; Escobedo, J. F.; Gomes, E. N., Pai, A. D. Global and diffuse solar irradiances in urban and rural areas in Southeast Brazil. Theoretical and Applied Climatology, v.93, p.57-73, 2008.

CPTEC - Centro de Previsão do Tempo e Estudos Climáticos/ INPE. Climanálise - Boletim de monitoramento e análise climática. <http://climanalise.cptec.inpe.br/ rclimanl/ boletim/>. 24 Jan. 2010.

Cunha, A. P.; Martins, D. Classificação climática para os municípios de Botucatu e São Manuel, SP. Irriga, v.14, p.111, 2009.

Escobedo, J; Gomes, E. N.; Oliveira, A; Soares, J. Modeling hourly and daily fractions of UV, PAR and NIR to global solar radiation under various sky conditions at Botucatu, Brazil. Applied Energy, v.86, p.299-309, 2009.

Iqbal, M. An introduction to solar radiation. London: Academic Press, 1983. 390p.

Kaufman, Y. J.; Koren, I. Smoke and pollution aerosol effect on cloud cover. Science, v.313, p.655-658, 2006.

Koren, I.; Martins, J. V.; Remer, L. A.; Afargan, H. Smoke invigoration versus inhibition of clouds over the Amazon. Science, v.321, p.946-949, 2008.

Kudish, A. I.; Ianetz, A. Analysis of daily clearness index, global and beam radiation for beer sheva, Israel: Partition according to day type and statistical analysis. Energy Conversion and Management, v.37, p.405-416, 1996.

Kvalevag, M. M.; Myhre, G. Human impact on direct and diffuse solar radiation during the industrial era. Jornal of Climate, v.20, p.4874-4883, 2007.

Li, D. H. W; Lam, J. C. An analysis of climatic parameters and sky condition classification. Building and Environment, v.36, p.435-450, 2000.

Li, D. H. W. ; Tang, H. L.; Cheung, K. L.; Lee, E. W. M.; Cheng, C. C. K. Sensitivity analysis of climatic parameters for sky classification. Theoretical and Applied Climatology, v.105, p.297-309, 2011.

Lohmann, U.; Feichter, J. Global indirect aerosol effects: a review. Atmospheric Chemistry and Physics, v.5, p.715-737, 2005.

Luo, Y; LÜ, D.; He, Q.; Li, W.; Zhou, X. Characteristics of atmospheric aerosol optical depth variation over China in recent 30 years. Chinese Science Bulletin, v.45, p.1328-1333, 2000.

NASA - National Aeronautics and Space Administration. Terra: the EOS Flagship. < http://terra.nasa.gov/>. 13 Fev. 2010.

Ogunjobi, K. O.; Kim, Y. J.; Adedokun, J. A.; Ryu, S. Y.; Kim, J. E. Analysis of sky conditions using solar radiation data at Kwangju and Seoul, South Korea and Ile-Ife, Nigeria. Theoretical and Applied Climatology, v.72, p.265-272, 2002. 
Okogbue, E. C.; Adedokun, J. A.; Holmgren, B. Hourly and daily clearness index and diffuse fraction at a tropical station, Ile-Ife, Nigéria. International Journal of Climatology, v.29, p.1035-1047, 2009.

Pattanasethanon, S.; Lertsatitthanakorn, C.; Atthajariyakul, S.; Soponronnarit, S. All sky modeling daylight availability and illuminance/irradiance on horizontal plane for Mahasarakham, Thailand. Energy Conversion \& Management, v.48, p.1601-1614, 2007.

Perez, R.; Ineichen, P.; Seals, R. Modeling daylight availability and irradiance components from direct and global irradiance. Solar Energy, v.44, p.271-289, 1990.
Reboita, M. S.; Gan, M. A.; Rocha, R. P.; Ambrizzi, T. Regimes de precipitação na América do Sul: Uma revisão bibliográfica. Revista Brasileira de Meteorologia, v.25, p.185-204, 2010.

Shapiro, S. S.; Wilk, M. B. An analysis of variance test for normality (complete samples). Biometika, v.52, p.591-611, 1965.

Souza, R. V. G. ; Robledo, L.; Pereira, F. O. R.; Soler, A. Evaluation of global luminous efficacy models for Florianópolis, Brazil. Building and Environment, v.41, p.1364-1371, 2006.

Wielicki, B. A., Wong, T., Loeb, N., Minnis, P., Priestley, K., Kandel, R. Changes in earth's albedo measured by satellite. Science, v.308, p.825-825, 2005. 